\title{
Short-Term Prognosis Value of sST2 for an Unfavorable Outcome in Hypertensive Patients
}

\author{
Anca Daniela Farcaş $\mathbb{D D}^{1,2}$ Mihaela Mocan ${ }^{10},{ }^{1,3}$ Florin Petru Anton $\mathbb{D}^{1,2}$ \\ Mocan-Hognogi Larisa Diana $\mathbb{D}^{1,2}$ Roxana Mihaela Chiorescu, ${ }^{1,3}$ Mirela Anca Stoia, ${ }^{1,2}$ \\ Camelia Larisa Vonica, ${ }^{4}$ Cerasela Mihaela Goidescu ${ }^{D},{ }^{1}$ and Luminița Animarie Vida-Simiti ${ }^{1}$ \\ ${ }^{1}$ Department of Internal Medicine, "Iuliu Hatieganu" University of Medicine and Pharmacy, Cluj-Napoca, Romania \\ ${ }^{2}$ Department of Cardiology, Emergency Clinical County Hospital, Cluj-Napoca, Romania \\ ${ }^{3}$ Department of Internal Medicine, Emergency Clinical County Hospital, Cluj-Napoca, Romania \\ ${ }^{4}$ Diabetes, Nutrition, and Metabolic Diseases Clinic, Emergency Clinical County Hospital, Cluj-Napoca, Romania
}

Correspondence should be addressed to Mihaela Mocan; mihaela.mocan@gmail.com

Received 7 August 2019; Revised 18 December 2019; Accepted 6 January 2020; Published 6 February 2020

Guest Editor: Ioana Mozos

\begin{abstract}
Copyright (c) 2020 Anca Daniela Farcaş et al. This is an open access article distributed under the Creative Commons Attribution License, which permits unrestricted use, distribution, and reproduction in any medium, provided the original work is properly cited.
\end{abstract}

\begin{abstract}
Background. sST2 represents a useful biomarker for the diagnosis and prognosis of patients with heart failure, but limited data is available on its role in patients with hypertension. The aim of this study is to evaluate the short-term prognosis value of sST2 for an unfavorable outcome in hypertensive patients. Methods. This was a prospective observational study which enrolled 80 patients with hypertension, who were followed for one year. All patients underwent clinical, laboratory (including sST2), and echocardiographic assessment at baseline. The patients were grouped according to the cardiovascular (CV) events reported during the follow-up: group A (with CV events) and group B (without CV events). Results. Overall, 59 CV events were reported during the follow-up period. Compared to group B, the patients in group A had significantly higher sST2 levels, a higher number of $\mathrm{CV}$ risk factors, and a higher left ventricle mass. Except for the diastolic dysfunction parameters, the echocardiographic findings were similar in the two groups. Patients in group A had a lower E/A ratio, larger deceleration time, and increased telediastolic pressure as quantified by the $E / E^{\prime}$ ratio than those in group B. Multivariate logistic regression analysis showed that sST2 and fasting plasma glucose at baseline were independent predictors for the CV events reported during the follow-up period. sST2 levels $>28.5 \mathrm{ng} / \mathrm{mL}$ were associated with poor clinical outcomes $(p=0.006$, Kaplan-Meier analysis). Conclusions. sST2 levels were correlated with the risk of adverse CV outcomes in hypertensive patients and may represent a useful prognostic marker in these patients.
\end{abstract}

\section{Introduction}

The receptor of suppression of tumorigenicity 2 (ST2) (also known as IL-1R4, DER4, Fit-1, or T1) is a type 1 transmembrane protein encoded by the IL-1RL1 gene, and the symbol for ST2 is approved by the Human Gene Nomenclature Database [1]. The gene is located on chromosome 2.12. The protein product of the ST2 gene encodes three isoforms identified in human tissues: a released soluble form (sST2, acting as a decoy receptor for IL-33, inhibiting IL-33/ST2L signal- ling) which can be detected in human serum, a transmembrane receptor (ST2L or ST2) discovered to be IL-33 in 2005, and a variant of ST2 (ST2V) [2].

Serum levels of sST2 are increased in conditions of ventricular biomechanical overload such as acute myocardial infarction (AMI) and proved to be useful in predicting mortality and heart failure (HF) in these patients [3]. Moreover, sST2 levels predict an outcome in patients with HF, and a variation of SST2 concentration over time is associated with prognosis [4]. Several other studies showed a correlation 
between levels of sST2 and the severity of HF, the left ventricular (LV) systolic function associated with valvular diseases [5], renal impairment, and other biomarkers of cardiac dysfunction such as B-type natriuretic peptide and C-reactive protein $[6,7]$.

Such data suggest that measurement of serum levels of sST2 may provide insight into the hemodynamic burden of the myocardium and might be useful for the early detection of cardiac disease, both systolic and diastolic. Taking these into consideration, investigating sST2 in patients with high blood pressure (HBP) was a logical stepwise approach as HBP affects the myocardium leading to left ventricular hypertrophy (LVH), left ventricular diastolic dysfunction (LVDD), and heart failure with preserved ejection fraction (HFpEF) $[8,9]$. Actually, hypertension is the most important risk factor for $\mathrm{HFpEF}$, with $75 \%$ of $\mathrm{HFpEF}$ patients being hypertensive [10]. The diagnosis of different pathogenic stages leading to $\mathrm{HFpEF}$ in hypertensive patients is based on ECG, which has low sensitivity but high specificity. Echocardiography, on the other hand, even though it is a sensitive and specific tool, lacks great accessibility, requires qualified personnel, and has low accuracy in obese or respiratory patients [9]. In the light of these findings, the identification of biomarkers useful for the early diagnosis and for prognosis of LVH, LVDD, and HFpEF in hypertensive patients is imperative and the data regarding this subject are scarce.

Thus, the present study was aimed at analyzing the relationship between serum levels of sST2 and the presence of LVH and LVDD in hypertensive patients, assessing at the same time the potential short-term prognosis value off sST2 for cardiovascular $(\mathrm{CV})$ events in these patients.

\section{Patients and Methods}

2.1. Study Population. 80 hypertensive patients (mean age $54.7 \pm 13.5$ years; $47.5 \%$ men and $52.5 \%$ women) were enrolled in a prospective observational study and followed for 1 year. The diagnosis of HBP was established according to the recommendations of the ESH/ESC guidelines [11]: $\mathrm{SBP} \geq 140 \mathrm{mmHg}$ and/or $\mathrm{DBP} \geq 90 \mathrm{mmHg}$ or if patients received treatment for HBP. Patients with chronic inflammatory or acute infectious diseases, heart disease (ischemic, congenital, and valvular heart disease, myocarditis, severe acute or chronic heart failure, and acute coronary syndrome), or pulmonary disease (COPD, asthma, and sleep apnea) were excluded. Clinical examination was performed, and demographic data, risk factors, and previous medical history were collected for all patients. Upon enrolment, all patients signed an informed consent and respected national and international legislation regarding clinical studies $[12,13]$.

2.2. Study Protocol. At baseline, the patients were examined clinically and fasting blood was collected by venous puncture, in the morning, after a rest of 5-10 minutes. Serum was obtained by centrifuging the coagulated blood for 15 minutes at $1000 \times g$ and stored at $-20^{\circ} \mathrm{C}$ until ST2 measurement, performed with the soluble ST2/IL-1R4 (human) and ELISA
Tecan Sunrise reader. For the ST2 assay, the analytical limit of detection (sensitivity) was $5 \mathrm{pg} / \mathrm{mL}$, intra-assay coefficients of variation (\%) were $4-6 \%$, and interassay coefficients of variation (\%) were $8-10 \%$.

Echocardiography was performed by an experienced sonographer using the $4-2 \mathrm{MHz}$ probe on a Philips Affiniti 50 machine and measured the parameters for cardiac remodeling and diastolic and systolic function. The dimensions of the walls and heart cavities were measured in the M-mode, and the ejection fraction was estimated using Simpson's biplane method. The LV mass was determined using the modified Devereaux's formula [11]: values $>95 \mathrm{~g} / \mathrm{m}^{2}$ in women and $>115 \mathrm{~g} / \mathrm{m}^{2}$ in men were considered diagnostic for the presence of LV hypertrophy.

Diastolic function was assessed using mitral diastolic flow parameters ( $A$ wave, $E$ wave, $E / A$ ratio, $E$-wave DT, and IVRT) and mitral annular (septal and lateral) tissue Doppler-mean value of $e^{\prime}\left(e^{\prime} \mathrm{m}\right)$ and $E / e(\mathrm{~m})$. Patients were followed for 1 year, and all $\mathrm{CV}$ events, such as hypertension emergencies, episodes of LV failure, and unstable angina, were registered. Hypertensive emergencies were defined as severe hypertension $(\geq 180 / 110 \mathrm{mmHg})$ in patients presenting to the emergency department in whom there is no clinical evidence of acute organ damage, in accordance with ESC Guidelines [11]. LV failure was considered in the presence of cardiac failure clinical signs (more than 2 symptoms from the Framingham score) and echocardiographic signs of diastolic and/or systolic LV dysfunction as described above. The diagnosis of unstable angina was established in accordance with the current criteria of the ESC Guidelines [14]: retrosternal pain with characteristic angina (de novo, aggravated by effort, and occurring at an early stage after percutaneous or surgical myocardial revascularization), the presence of electrocardiographic changes, and the absence of a myocardial enzymatic reaction (CK, $\mathrm{CK}-\mathrm{MB}$, and troponin). The following ECG changes were considered diagnosis criteria for unstable angina: ST depression $\geq 0.05 \mathrm{mV}$ in two or more contiguous leads, ST depression combined with transient ST elevation (not fulfilling the STEMI criteria mentioned above), and T-wave inversion [14]. The ST and/or T variability on different ECG was considered another important diagnosis criterion for unstable angina.

2.3. Statistical Analysis. Statistical analyses were performed with IBM $^{\circledR}$ SPSS $^{\circledR}$ package version 19 (IBM Corporation, Armonk, NY, USA). Normally distributed continuous variables were expressed as mean and standard deviation and compared using the Student test. Continuous variables with abnormal distribution and ordinal variables were compared using the Mann-Whitney $U$ test. Categorical variables were expressed as numbers and percentages, and the group comparison was done using the $\chi^{2}$ test. Correlation coefficients were calculated by linear regression analysis, while multiple regression analysis was applied for analysis of the dependency between variables. $p<0.05$ was considered statistically significant. The predictive capacity of ST2 was evaluated using computed areas under the receiver operating curve (AUC). A value of $p<0.05$ was deemed significant; confidence intervals (CI) were calculated for $p=0.05$ as the threshold. 
TABLE 1: Baseline characteristics of all patients, group A, and group B.

\begin{tabular}{|c|c|c|c|c|c|}
\hline Characteristics & All patients $80(\%)$ & & $\begin{array}{l}\text { Group A (HBP with CV events) } \\
36 \text { patients }\end{array}$ & $\begin{array}{l}\text { Group B (HBP without CV events) } \\
44 \text { patients }\end{array}$ & $p$ \\
\hline Age & $54.7 \pm 13.5$ & & $55.23 \pm 14.47$ & $52.68 \pm 11.53$ & 0.06 \\
\hline \multicolumn{6}{|l|}{ Sex } \\
\hline Men & $38(47.5 \%)$ & 0.051 & $18(50 \%)$ & $20(45.45 \%)$ & 0.061 \\
\hline Women & $42(52.5 \%)$ & & $18(50 \%)$ & $24(54.54 \%)$ & 0.052 \\
\hline \multicolumn{6}{|l|}{ Hypertension (HBP) } \\
\hline Stage I & $20(25 \%)$ & $<0.001^{\mathrm{a}}$ & $10(27.77 \%)$ & $10(22.72 \%)$ & 0.028 \\
\hline Stage II & $34(42.5 \%)$ & $0.002^{\mathrm{b}}$ & $12(27.77 \%)$ & $22(50 \%)$ & 0.001 \\
\hline Stage III & $26(32.5 \%)$ & $0.023^{\mathrm{c}}$ & $14(38.88 \%)$ & $12(27.27 \%)$ & 0.005 \\
\hline \multicolumn{6}{|l|}{$\begin{array}{l}\text { Additional cardiovascular } \\
\text { risk }\end{array}$} \\
\hline Moderate & $15(18.75 \%)$ & $0.002^{\mathrm{a}}$ & $3(8.34 \%)$ & $12(27.27 \%)$ & 0.03 \\
\hline High & $33(41.25 \%)$ & $0.023^{\mathrm{b}}$ & $12(33.3 \%)$ & $21(47.72 \%)$ & $0.008^{\mathrm{b}}$ \\
\hline Very high & $21(26.25 \%)$ & $<0.001^{\mathrm{c}}$ & & $11(25 \%)$ & $0.000^{\mathrm{c}}$ \\
\hline \multicolumn{6}{|l|}{ Controlled hypertension } \\
\hline No & $25(31.25 \%)$ & & $21(58.33 \%)$ & $4(9.09 \%)$ & $<0.001$ \\
\hline Yes & $55(68.75 \%)$ & $<0.001$ & $15(41.66 \%)$ & $40(90.90 \%)$ & $<0.001$ \\
\hline \multicolumn{6}{|l|}{ Dyslipidaemia } \\
\hline Without & $8(10 \%)$ & & $3(8.33 \%)$ & $5(11.36 \%)$ & 0.04 \\
\hline Hypercholesterolemia & $42(52.5 \%)$ & & $22(61.11 \%)$ & $20(45.45 \%)$ & 0.002 \\
\hline Mixed & $26(32.5 \%)$ & & $11(30.55 \%)$ & $15(34.09 \%)$ & 0.05 \\
\hline Hypertriglyceridemia & $4(5 \%)$ & & - & $4(9.1 \%)$ & - \\
\hline \multicolumn{6}{|l|}{ Smoke } \\
\hline No & $58(72.5 \%)$ & & $18(50 \%)$ & $40(90.9 \%)$ & 0.001 \\
\hline Yes & $22(27.5 \%)$ & 0.001 & $18(50 \%)$ & $4(9.1 \%)$ & 0.001 \\
\hline \multicolumn{6}{|l|}{ BMI } \\
\hline Normal weight & $32(40 \%)$ & $0.04^{\mathrm{a}}$ & $5(13.88 \%)$ & $27(61.63 \%)$ & 0.003 \\
\hline Overweight & $28(35 \%)$ & $0.45^{\mathrm{b}}$ & $16(44.44 \%)$ & $12(27.27 \%)$ & 0.026 \\
\hline Obese & $20(25 \%)$ & $0.03^{c}$ & $15(41.66 \%)$ & $5(11.36 \%)$ & 0.018 \\
\hline \multicolumn{6}{|l|}{ Diabetes mellitus (DM) } \\
\hline No & $54(67.5 \%)$ & & $20(55.55 \%)$ & $34(77.27 \%)$ & 0.021 \\
\hline Yes & $26(32.5 \%)$ & 0.001 & $16(44.44 \%)$ & $10(22.72 \%)$ & $<0.001$ \\
\hline
\end{tabular}

${ }^{\mathrm{a}}$ Comparisons between 1 and 2 ; ${ }^{\mathrm{b}}$ comparisons between 2 and $3 ;{ }^{\mathrm{c}}$ comparisons between 1 and 3.

\section{Results}

Baseline clinical and demographic characteristics of the 80 patients are shown in Table $1.30 \%$ of the patients had grade $3 \mathrm{HBP}$ and $45 \%$ had grade $2 \mathrm{HBP}$. In $68.75 \%$ of the patients, $\mathrm{BP}$ values were correctly controlled at the first examination, in accordance with the recommendations of the European guidelines [15]. Patients had several modifiable or nonmodifiable risk factors. Dyslipidaemia was found in $90 \%$ of the patients (hypercholesterolemia in $52.5 \%$ of them, mixed in $32.5 \%$ of them, and hypertriglyceridemia in $5 \%$ of them). $27.5 \%$ of the patients were smokers, and $51.25 \%$ of them had weight problems $(27.5 \%$ were overweight and $25 \%$ obese). Diabetes was associated with HBP in 35\% of cases. During the one-year study follow-up, 36 patients (45\%) presented a total number of $59 \mathrm{CV}$ events. Patients with $\mathrm{CV}$ events were included in group A (36 patients), and those without were included in group B (44 patients).

Table 1 shows the characteristics of the patients in the two groups. There was no significant difference regarding gender and age of the patients, but patients in group A had a higher proportion of stage $3 \mathrm{HBP}$ with very high additional $\mathrm{CV}$ risk (e.g., 10-year cardiovascular risk categories using European guideline recommendations for hypertension [15]) and worse BP control rates than those in group B. In group $\mathrm{A}$, we found more patients presenting dyslipidaemia (with hypercholesterolemia and mixed dyslipidaemia), smokers with higher BMI (overweight and obese), and diabetics compared with group B. Serum sST2 levels were higher in patients in group A compared to group B.

There were no significant differences between the two groups except for the parameters of diastolic dysfunction. 
TABLE 2: Clinical, laboratory, and echocardiographic data of patients in the two groups.

\begin{tabular}{|c|c|c|c|}
\hline Variables & $\begin{array}{c}\text { Group A (HBP with CV events) } \\
36 \text { patients }\end{array}$ & $\begin{array}{l}\text { Group B (HBP without CV events) } \\
44 \text { patients }\end{array}$ & $p$ \\
\hline Age & $55.23 \pm 14.47$ & $52.68 \pm 11.53$ & 0.06 \\
\hline Men & $18(50 \%)$ & $20(45.45 \%)$ & 0.061 \\
\hline Women & $18(50 \%)$ & $24(54.54 \%)$ & 0.052 \\
\hline SBP (mmHg) & $156.00 \pm 24.56$ & $136.63 \pm 26.71$ & 0.023 \\
\hline $\mathrm{DBP}(\mathrm{mmHg})$ & $95.22 \pm 28.61$ & $84.76 \pm 12.92$ & 0.024 \\
\hline BMI $\left(\mathrm{kg} / \mathrm{m}^{2}\right)$ & $32.42 \pm 6.71$ & $27.84 \pm 7.11$ & 0.03 \\
\hline Uric acid (mg/dL) & $6.75 \pm 1.65$ & $4.98 \pm 2.13$ & 0.079 \\
\hline Creatinine (mg/dL) & $0.9 \pm 0.51$ & $0.79 \pm 0.38$ & 0.246 \\
\hline Serum glucose $(\mathrm{mg} / \mathrm{dL})$ & $110.55 \pm 31.64$ & $104.36 \pm 33.78$ & 0.338 \\
\hline Total cholesterol (mg/dL) & $160.72 \pm 86.81$ & $144.05 \pm 58.84$ & 0.57 \\
\hline HDL cholesterol (mg/dL) & $47.05 \pm 8.94$ & $48.59 \pm 13.46$ & 0.48 \\
\hline LDL cholesterol (mg/dL) & $104.81 \pm 49$ & $103.97 \pm 52.88$ & 0.49 \\
\hline Triglycerides (mg/dL) & $128.70 \pm 87.53$ & $119.58 \pm 83.52$ & 0.08 \\
\hline $\mathrm{ST} 2(\mathrm{ng} / \mathrm{mL})$ & $52.71(41.7-99.45)$ & $21.34(15.17-44.24)$ & 0.002 \\
\hline Ascending aorta (mm) & $30.92 \pm 3.90$ & $29.46 \pm 8.45$ & 0.002 \\
\hline Left atrium size $(\mathrm{mm})$ & $33.63 \pm 9.56$ & $34.04 \pm 5.71$ & 0.056 \\
\hline Left atrium area $(\mathrm{mm})$ & $10.65 \pm 5.39$ & $10.42 \pm 6.92$ & 0.053 \\
\hline End-systolic interventricular septum (mm) & $12.19 \pm 2.05$ & $12.00 \pm 2.26$ & 0.051 \\
\hline End-diastolic interventricular septum (mm) & $12.19 \pm 2.05$ & $12.00 \pm 2.26$ & 0.188 \\
\hline End-systolic LV posterior wall (mm) & $13.07 \pm 5.14$ & $12.99 \pm 5.344$ & 0.051 \\
\hline End-diastolic LV posterior wall (mm) & $11.68 \pm 1.79$ & $11.33 \pm 2.64$ & 0.052 \\
\hline End-systolic LV size (mm) & $27.73 \pm 12.29$ & $27.04 \pm 11.97$ & 0.224 \\
\hline End-diastolic LV size (mm) & $45.26 \pm 6.04$ & $42.36 \pm 9.89$ & 0.306 \\
\hline Right ventricle size (mm) & $27.19 \pm 7.08$ & $25.14 \pm 9.16$ & 0.074 \\
\hline Stroke volume $(\mathrm{mL})$ & $34.83 \pm 12.86$ & $32.60 \pm 11.70$ & 0.001 \\
\hline LV mass $\left(\mathrm{g} / \mathrm{m}^{2}\right)$ & $176.4 \pm 26.2$ & $155.13 \pm 83.6$ & $<0.001$ \\
\hline LV mass index & $146.95 \pm 75.80$ & $140.15 \pm 82.93$ & $<0.001$ \\
\hline IVRT (ms) & $112.12 \pm 46.7$ & $104.56 \pm 57.03$ & 0.009 \\
\hline$E / A$ & $0.78 \pm 0.43$ & $0.97 \pm 0.59$ & 0.0043 \\
\hline $\mathrm{EDT}(\mathrm{ms})$ & $225.86 \pm 75.06$ & $204.64 \pm 99.24$ & 0.0032 \\
\hline$E / E^{\prime} \mathrm{m}$ & $10.64 \pm 2.33$ & $8.24 \pm 3.56$ & 0.0035 \\
\hline
\end{tabular}

Patients in group $\mathrm{A}$ had a lower $E / A$ ratio, a longer $E$-wave DT, and significantly higher end-diastolic pressure (quantified by the $E^{\prime} / E$ ratio) (Table 2).

Admission sST2 levels were significantly correlated with CV events $(p<0.001)$. Table 3 shows the univariate association between CV events and log-transformed sST2 and clinical or echocardiographic parameters. CV event risk increases with increasing sST2 levels and glycemia. $80.7 \%$ of CV event number variability is determined by the sST2 level and glycemia. Regression analysis showed that $80.7 \%$ of CV event number variability can be explained by a $10 \mathrm{ng} / \mathrm{mL}$ increase in sST2 level and a $10 \mathrm{mg} / \mathrm{dL}$ increase in glycemia.

Diastolic function parameters $-E / A$ ratio and $E / e m$ ratio-and LV mass were correlated with the incidence and number of CV events. Multivariate analysis showed that sST2 and fasting glucose independently increased the risk of $\mathrm{CV}$ events over a period of 1 year of follow-up (Table 4).

The receiver operating characteristic curve (ROC) for sST2 concentration identified $28.5 \mathrm{ng} / \mathrm{mL}$ as the optimal cut-off value to predict CV events with sensitivity and specificity of $94.4 \%$ and $69.1 \%$, respectively $(p=0.000)$. An area under the ROC curve (AUC) was 0.84 (95\% CI 0.78-0.89) which indicates the discriminative potential of this value of sST2 between high- and low-risk patients. Kaplan-Meier curve analysis showed that patients with sST2 $>28.5 \mathrm{ng} / \mathrm{mL}$ had a higher occurrence of CV events (HR 2.43, $p<0.001$ ) (Figures 1 and 2). 
TABLE 3: Univariate and multivariate analyses showing association between CV events and log-transformed ST2.

\begin{tabular}{lcccc}
\hline & \multicolumn{2}{c}{$\begin{array}{c}\text { Univariate analysis } \\
\text { Coefficient of } \\
\text { correlation }\end{array}$} & $p$ & \multicolumn{3}{c}{$\begin{array}{c}\text { Multivariate analysis } \\
\text { Coefficient of } \\
\text { correlation }\end{array}$} & $p$ \\
\hline ST2 & 0.696 & $<0.001$ & 0.64 & 0.002 \\
Fasting glucose & 0.380 & 0.020 & 0.34 & 0.0032 \\
$\mathrm{LV}$ mass $\left(\mathrm{g} / \mathrm{m}^{2}\right)$ & 0.44 & $<0.001$ & 0.38 & 0.051 \\
$\mathrm{E} / \mathrm{A}$ & 0.28 & 0.027 & 0.34 & 0.07 \\
$\mathrm{E} / \mathrm{e}^{\prime} \mathrm{m}$ & 0.31 & 0.021 & 0.45 & 0.06 \\
$\mathrm{TEj}$ & 0.289 & 0.006 & & \\
\hline
\end{tabular}

TABLE 4: Multivariate analysis showing sST2 and fasting glucose independent correlation with $\mathrm{CV}$ events on the short term.

\begin{tabular}{lcc}
\hline Variable & $\begin{array}{c}\text { Hazard ratio }(95 \% \mathrm{CI}) \\
\text { CV events }\end{array}$ & $p$ \\
\hline sST2 & $2.43(1.32-7.24)$ & 0.005 \\
Fasting glucose & $1.43(1.041-1.732)$ & 0.0023 \\
\hline
\end{tabular}

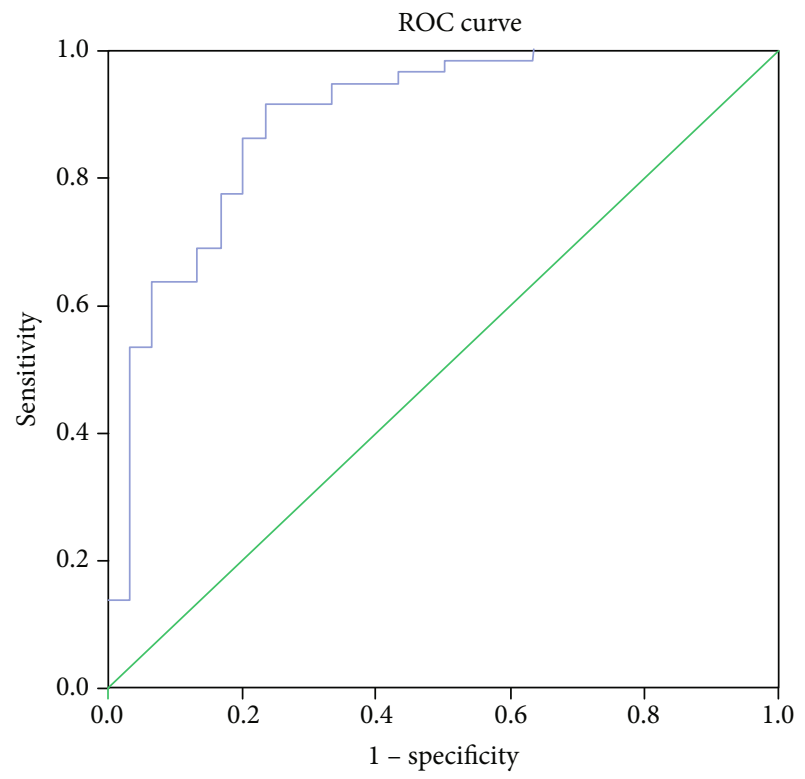

FIGURE 1: ROC analysis showing the sST2 sensitivity and specificity for predicting the $\mathrm{CV}$ events during one year after hospitalization.

\section{Discussions}

Our study showed that sST2 levels are higher in hypertensive patients with CV events than in those free of CV events. sST2 levels are correlated with higher LVM, a number of CV risk factors, and the presence of LVDD. Fasting glucose and sST2 are correlated with CV events on the short term.

4.1. sST2, HBP, and LVDD. Other small studies of sST2 variations were performed in hypertensive patients, with similar results. Ojji et al. in 2013 suggested a link between LV geometry and sST2. The authors raised the hypothesis that sST2

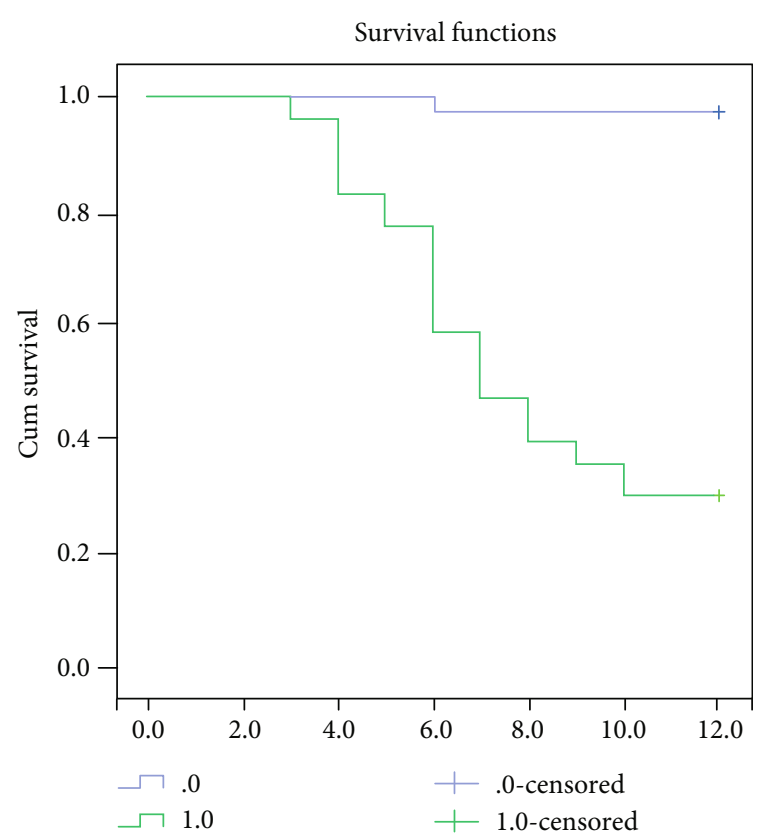

FIgURE 2: Kaplan-Meier curve analysis revealed that patients with $\mathrm{sST} 2>28.5 \mathrm{ng} / \mathrm{mL}$ had a higher occurrence of CV events.

level was not only affected by hypertensive LVH but might be a future biomarker in differentiating concentric hypertrophy from normal geometry in HBP [16]. The study comprised 133 consecutive patients diagnosed with HBP, with $37 \%$ presenting LVH [16]. Later on, the same group showed that sST2 serum levels correlated strongly with clinical and echocardiographic parameters and correlated well with NTpro-BNP [17]. So sST2 might be useful to distinguish between hypertensive patients with and without LVH. Our group (Farcas et al.) has previously shown that sST2 could be useful as an early diagnostic biomarker for cardiac remodeling and altered diastolic performance in HBP, providing additional data to echocardiography. It could represent a milestone in early detection of cardiac performance alteration [18]. Furthermore, Wang et al. performed a larger study on 344 patients with HBP and HFpEF and showed that sST2 measurement provides diagnostic aid of stable HFpEF, correlated with NYHA class and LVDD [19]. In these patients, combined measurement provided an advanced risk stratification value compared to one biomarker measurement alone [19]. As a physiopathological explanation of the link between altered diastolic performance and high sST2 levels in hypertensive patients, Bartunek et al. showed that in humans with cardiac hypertrophy and heart failure, serum sST2 correlates with the diastolic load and has an extramyocardial source [20]. Furthermore, Zile et al. discovered that $\mathrm{HBP}$ in the presence of HFpEF alters passive myocardial stiffness with simultaneous increase in inflammation and fibrosis biomarkers, such as sST2, sustaining the hypothesis that the development of HFpEF depends on changes in both collagen and titin homeostasis [21].

4.2. sST2 and Short-Term Outcomes. In our study, we found that sST2 correlated with short-term prognosis for CV events 
in hypertensive patients. Released as a consequence of myocardial strain, influenced by inflammation and imbalance of the extracellular matrix, sST2 may be a suitable biomarker for prognosis, i.e., LVDD progression to HF [22]. Most of the large studies focused on the prognosis value of sST2 in HFrEF [23-25] and in acute coronary syndromes [3], rather than in ambulatory patients. In a multicentric study of 447 HFpEF patients admitted for acute HF, the authors demonstrated a comparable prognostic value of sST2 in both HFpEF and HFrEF [26]. When evaluated in ambulatory patients with HF, sST2 provided "valuable long-term risk stratification information in HF beyond that reported by other biomarkers of stretch, inflammation, necrosis, and remodeling" [27].

4.3. sST2 Relation to Other Imaging Methods. One of the main findings in our study is the relations of sST2 levels and LVDD diagnosed by echocardiography. The potential diagnosis capacity of sST2 in LVDD and its correlations with echocardiographic findings were elegantly reviewed by DeFilippi et al. [28]. In brief, large studies such as the Cardiovascular Health Study, comprising older patients ( $>65$ years old) with $L V D D \geq$ grade 1 present in $24.1 \%$, showed that sST2 was strongly associated with LVDD (OR 1.35 (95\% confidence interval 1.06-1.72)) and especially with echocardiographic criteria ( $E / A$ mitral inflow) and had the capacity to improve the diagnosis accuracy [29]. On the other hand, the Framingham study showed no correlation between sST2 and echocardiographic criteria for LVDD, supporting the idea that sST2 might be more suitable for a risk screening strategy in large cohorts, rather than a screening tool for structural heart disease [30]. Moreover, Daniels et al. evaluated 588 ambulatory patients with HF and found that sST2 was predominantly associated with right ventricle and not LV structural alterations [31]. The question remains whether this strong association between sST2 and LVDD in elders is a result of a cardiac-specific effect or it is influenced by general factors such as inflammation or vascular stiffness, which are common findings in aging patients [28].

4.4. sST2 in comparison to Other Biomarkers. Our study focused on single-marker evaluation, rather than on a multimarket strategy, for the diagnosis of LVDD. In our previous work, we assessed the potential of N-terminal pro-B-type natriuretic peptide as a diagnosis biomarker for LVDD in hypertensive patients with metabolic syndrome [32]. As in patients with metabolic syndrome, in our study group, 34\% of the patients were diagnosed with diabetes mellitus (DM) and fasting glucose, along with higher sST2 levels, is associated with higher risk of $\mathrm{CV}$ events. In this respect, Ruocco et al. showed that ST2 was higher in patients with LVDD and DM, it significantly correlated with glycosylated haemoglobin (HbA1c), and it was related to an adverse event occurrence within 6 months and with poor prognosis [33]. Miller et al. have recently demonstrated that sST2 is related to $\mathrm{DM}$ and inflammation rather than $\mathrm{CV}$ risk factors, blood pressure, or smoking [34]. The pathogenic link between circulating sST2 and DM is still not clear but could be causal. The authors hypothesized that sST2 not only is a biomarker but also may contribute to the pathogenesis of diabetes via IL-33 interactions. The IL-33/ST2 pathway may participate in the inflammatory and remodeling processes of various tissues in patients with DM [34]. The potential diagnosis biomarkers in cardiac remodeling after myocardial infarction in patients with HBP and DM were a subject of a previous study published by our group. We showed that lower leptin levels were associated with reduced values of echocardiographic parameters of ventricular remodeling [35]. Further research should focus on sST2 and cardiac remodeling after myocardial infarction.

Other studies showed sST2 to improve discrimination when adjusted to multivariable models comprising $\mathrm{N}$ terminal pro-B-type natriuretic peptide or galectin-3 [27]. sST2 as compared to other biomarkers has the advantage not to be influenced by confounders (renal function, BMI, or age), and its levels are modified by the progressing disease.

4.5. Limitations of the Study. There are some limitations to our study that are worth taking into consideration. First of all, our study is a cross-sectional study with a single measurement of biomarker levels and is limited by the small number of patients. As such, the study does not benefit from the inherent variability in time of the tested biomarkers. As outpatients were included, the study group consisted of rather young patients, equally distributed by sex. Thus, these results cannot necessarily be extrapolated to older populations. Also, the study population was not tested for underlying coronary artery disease, which could also present as LVDD. Moreover, the interaction between medical therapy and the serum levels of the studied biomarkers was not addressed in this analysis, which was more focused on the links between SST2 and echocardiographic parameters of LVDD. Finally, we have to acknowledge that the number of participants was relatively small because of the multiple exclusion criteria, but it was enough to give a study power of over 0.80 . Larger clinical trials will be needed for the validation of an ST2-predictive value in clinical practice.

\section{Conclusions}

Serum levels of sST2 are strongly correlated with higher CV risk in hypertensive patients and have a predictive potential for poor prognosis in these patients. Fasting glucose and sST2 are correlated with CV events on the short term.

\section{Data Availability}

The clinical data used to support the findings of this study are included within the article.

\section{Disclosure}

Parts of this manuscript were presented at the European Congress of Cardiology 2018 (Abstract P1866 European Heart Journal (2018) 39 (Supplement), 396).

\section{Conflicts of Interest}

The authors declare that they have no conflicts of interest. 


\section{Authors' Contributions}

Anca Daniela Farcaş, Mihaela Mocan, Florin Petru Anton, and Luminița Animarie Vida-Simiti contributed equally to this work. Anca Daniela Farcaş and Luminița Animarie Vida-Simiti designed the research study. Florin Petru Anton, Diana Larisa Mocan-Hognogi, Roxana Mihaela Chiorescu, and Mirela Anca Stoia performed the research. Anca Daniela Farcaş, Mihaela Mocan, Camelia Larisa Vonica, and Cerasela Mihaela Goidescu analyzed the data. Mihaela Mocan and Anca Daniela Farcaş wrote the paper.

\section{Acknowledgments}

The authors are grateful for the support of the Echocardiography Laboratory and of the Immunology Laboratory of Emergency County Hospital Cluj-Napoca that made the research possible.

\section{References}

[1] X. Liu, Y. Xiao, Y. Pan, H. Li, G. Zheng, and W. Su, "The role of the IL-33/ST2 axis in autoimmune disorders: friend or foe?," Cytokine \& Growth Factor Reviews, vol. 50, pp. 60-74, 2019.

[2] A. Dattagupta and S. Immaneni, "ST2: current status," Indian Heart Journal, vol. 70, pp. S96-S101, 2018.

[3] M. Shimpo, D. A. Morrow, E. O. Weinberg et al., "Serum levels of the interleukin-1 receptor family member ST2 predict mortality and clinical outcome in acute myocardial infarction," Circulation, vol. 109, no. 18, pp. 2186-2190, 2004.

[4] L. C. VarkVan, I. Lesman-leegte, S. J. Baart et al., "Prognostic Value of Serial ST2 Measurements in Patients With Acute Heart Failure," Journal of the American College of Cardiology, vol. 70, no. 19, pp. 2378-2388, 2017.

[5] I. Fabiani, L. Conte, N. Pugliese et al., "The integrated value of sST2 and global longitudinal strain in the early stratification of patients with severe aortic valve stenosis: a translational imaging approach," The International Journal of Cardiovascular Imaging, vol. 33, no. 12, pp. 1915-1920, 2017.

[6] W. Meijers, A. van der Velde, and R. DeBoer, "Biomarkers in heart failure with preserved ejection fraction," Netherlands Heart Journal, vol. 24, no. 4, pp. 252-258, 2016.

[7] M. Mocan, L. Diana, M. Hognogi et al., "Biomarkers of inflammation in left ventricular diastolic dysfunction," Disease Markers, vol. 2019, 14 pages, 2019.

[8] M. Santos and A. Shah, "Alterations in cardiac structure and function in hypertension," Current Hypertension Reports, vol. 16, no. 5, pp. 1-10, 2014.

[9] P. Palmiero, A. Zito, M. Maiello et al., "Left ventricular diastolic function in hypertension: methodological considerations and clinical implications," Journal of Clinical Medicine Research, vol. 7, no. 3, pp. 137-144, 2014.

[10] A. S. Go, D. Mozaffarian, V. L. Roger et al., "Heart Disease and Stroke Statistics-2013 Update," Circulation, vol. 127, no. 1, pp. e6-e245, 2013.

[11] H. Marwick, T. C. Gillebert, G. Aurigemma, and E. Al, "Recommendations on the use of echocardiography in adult hypertension: a report from the European Association of Cardiovascular Imaging (EACVI) and the American Society of Echocardiography (ASE) $\dagger$," European Heart Journal - Cardiovascular Imaging, vol. 16, pp. 577-605, 2015.
[12] S. I. Vida, "Interventional clinical and chemical studies in Romania, legal guarantees," Revista de Chimie, vol. 69, pp. 267-270, 2018.

[13] I. Vida-Simiti, "Medical liability for off label use of drugs in Romania," Revista de Chimie, vol. 68, pp. 755-758, 2018.

[14] M. Roffi, C. Patrono, and J. Collet, "2015 ESC Guidelines for the management of acute coronary syndromes in patients presenting without persistent ST-segment elevation," European Heart Journal, vol. 37, no. 3, pp. 267-315, 2016.

[15] G. Mancia, R. Fagard, K. Narkiewicz et al., "2013 ESH/ESC guidelines for the management of arterial hypertension: the Task Force for the management of arterial hypertension of the European Society of Hypertension (ESH) and of the European Society of Cardiology (ESC)," European Heart Journal, vol. 34, no. 28, pp. 2159-2219, 2013.

[16] D. B. Ojji, L. H. Opie, S. Lecour, L. Lacerda, O. Adeyemi, and K. Sliwa, "Relationship between left ventricular geometry and soluble ST2 in a cohort of hypertensive patients," Journal of Clinical Hypertension, vol. 15, no. 12, pp. 899-904, 2013.

[17] D. B. Ojji, L. H. Opie, S. Lecour, L. Lacerda, O. M. Adeyemi, and K. Sliwa, "The effect of left ventricular remodelling on soluble ST2 in a cohort of hypertensive subjects," Journal of Human Hypertension, vol. 28, no. 7, pp. 432-437, 2014.

[18] A. D. Farcas, F. P. Anton, C. M. Goidescu, I. L. Gavrilă, L. A. Vida-Simiti, and M. A. Stoia, "Serum soluble ST2 and diastolic dysfunction in hypertensive patients," Disease Markers, vol. 2017, 8 pages, 2017.

[19] Y. Wang, C. Yu, F. Chiu et al., "Soluble ST2 as a biomarker for detecting stable heart failure with a normal ejection fraction in hypertensive patients," Journal of Cardiac Failure, vol. 19, no. 3, pp. 163-168, 2013.

[20] J. Bartunek, L. Delrue, F. Van Durme et al., "Nonmyocardial production of ST2 protein in human hypertrophy and failure is related to diastolic load," Journal of the American College of Cardiology, vol. 52, no. 25, pp. 2166-2174, 2008.

[21] M. R. Zile, C. F. Baicu, J. S. Ikonomidis et al., "Myocardial stiffness in patients with heart failure and a preserved ejection fraction: contributions of collagen and titin," Circulation, vol. 131, no. 14, pp. 1247-1259, 2015.

[22] A. Bayés-Genís, J. Núñez, and J. Lupón, “Soluble ST2 for Prognosis and Monitoring in Heart Failure: The New Gold Standard?," Journal of the American College of Cardiology, vol. 70, no. 19, pp. 2389-2392, 2017.

[23] C. G. Bahuleyan, G. K. Alummoottil, J. Abdullakutty et al., "Prognostic value of soluble ST2 biomarker in heart failure patients with reduced ejection fraction - a multicenter study," Indian Heart Journal, vol. 70, pp. S79-S84, 2018.

[24] I. S. Anand, T. S. Rector, M. Kuskowski, J. Snider, and J. N. Cohn, "Prognostic value of soluble ST2 in the Valsartan Heart Failure Trial," Circulation: Heart Failure, vol. 7, no. 3, pp. 418426, 2014.

[25] J. L. Januzzi, A. Mebazaa, and S. Disomma, "ST2 and prognosis in acutely decompensated heart failure: the International ST2 Consensus Panel," The American Journal of Cardiology, vol. 115, no. 7, pp. 26B-31B, 2015.

[26] S. Manzano-Fernndez, T. Mueller, D. Pascual-Figal, Q. A. Truong, and J. L. Januzzi, "Usefulness of soluble concentrations of interleukin family member ST2 as predictor of mortality in patients with acutely decompensated heart failure relative to left ventricular ejection fraction," The American Journal of Cardiology, vol. 107, no. 2, pp. 259-267, 2011. 
[27] A. Bayes-Genis, J. Januzzi, H. Gaggin et al., "ST2 pathogenetic profile in ambulatory heart failure patients," Journal of Cardiac Failure, vol. 21, no. 4, pp. 355-361, 2014.

[28] C. DeFilippi, L. B. Daniels, and A. Bayes-Genis, "Structural Heart Disease and ST2: Cross-Sectional and Longitudinal Associations With Echocardiography," The American Journal of Cardiology, vol. 115, no. 7, pp. 59B-63B, 2015.

[29] S. Seliger, E. Ginsberg, J. Gottdiener, R. Christenson, and C. DeFilippi, "Soluble ST2 and galectin-3 are associated with subclinical diastolic dysfunction in older adults," Journal of the American College of Cardiology, vol. 63, no. 12, p. A769, 2014.

[30] T. J. Wang, K. C. Wollert, M. G. Larson et al., "Prognostic utility of novel biomarkers of cardiovascular stress: the Framingham Heart Study," Circulation, vol. 126, no. 13, pp. 1596-1604, 2012.

[31] L. Daniels, P. Clopton, N. Iqbal, K. Tran, and A. Maisel, "Association of ST2 levels with cardiac structure and function and mortality in outpatients," American Heart Journal, vol. 160, no. 4, pp. 721-728, 2010.

[32] M. Mocan, F. Anton, S. Suciu, R. Rahaian, S. N. Blaga, and A. D. Farcas, "Multimarker assessment of diastolic dysfunction in metabolic syndrome patients," Metabolic Syndrome and Related Disorders, vol. 15, no. 10, pp. 507-514, 2017.

[33] G. Ruocco, I. Evangelista, B. Franci et al., "Combination of ST2 and B-type natriuretic peptide in diabetic patients with acute heart failure," Journal of Cardiovascular Medicine, vol. 20, no. 2, pp. 81-90, 2019.

[34] A. M. Miller, D. Purves, A. Mcconnachie et al., "Soluble ST2 Associates with Diabetes but Not Established Cardiovascular Risk Factors: A New Inflammatory Pathway of Relevance to Diabetes?," PLoS One, vol. 7, no. 10, article e47830, 2012.

[35] A. D. Farcas, A. Rusu, M. A. Stoia, and L. A. Vida-Simiti, "Plasma leptin, but not resistin, TNF- $\alpha$ and adiponectin, is associated with echocardiographic parameters of cardiac remodeling in patients with coronary artery disease," Cytokine, vol. 103, pp. 46-49, 2018. 